\title{
Foin du passéisme!
}

La santé est à la mode: les deux grands partis du centre-droit de notre éventail politique national ont chacun tenu, il y a dix jours, une Assemblée des délégués lors de laquelle une grande part de l'ordre du jour a été consacrée au système de santé.

On y a entendu beaucoup de discours polémiques mais rien qui puisse, hélas, tirer enfin notre système de santé vers des rivages synonymes de solutions; il fut impressionnant une fois de plus de voir à quel point des idées caduques, connues pour être dépassées et inefficaces, servent de fonds de commerce à certaines personnalités - on aurait envie de leur proposer d'oser une fois toucher à leur "powerpoint»...

Dommage, en tout cas, d'en rester à de vieilles lunes alors que le corps médical, par la voix de la FMH en particulier, s'attache à proposer des solutions novatrices et constructives sur quantité de questions. Quelques exemples?

- Le corps médical a fait des efforts remarquables pendant toute la période de neutralité des coûts, à la suite de l'introduction du Tarmed; ces efforts, illustrant la capacité des médecins à obtenir les résultats attendus même dans une situation difficile, sont de très bon augure pour la suite: les Contrats Prestations et Coûts qui sont en cours de négociation dans les cantons pour «succéder» à la neutralité des coûts partent sur de bonnes bases.

Est-ce parce que cela démontre le sens civique et la bonne volonté des médecins, et parce que cela dément la rengaine des médecins irresponsables, que le Chef du Département de l'Intérieur n'a pu s'empêcher, l'autre samedi, de persifler les solutions partenariales?

- La FMH a fait tout une série de propositions pour répondre aux soucis du monde politique quant à la qualité des prestations médicales ou à l'économicité des prises en charge: nous avons dit il y a déjà un an que pour nous médecins, si le droit à un contrat doit rester automatique, cet automatisme peut être soumis à des conditions qui tant vis-à-vis du monde politique que de l'administration que du public, assurent que les insuffisances et les abus seront efficacement bannis, et les coûts maîtrisés.

Ces propositions, partenariales de nouveau et qui sont de vraies réponses à des soucis réels, permettent de dépasser le débat stérile sur l'abolition du libre choix du médecin, qui aboutirait immanquablement à un référendum.
La Commission de la santé et de la sécurité sociale du Conseil des Etats a bien compris, enfin, qu'il y avait là une occasion à saisir (communiqué du 30 août 2005 sur le financement des hôpitaux): le projet de la Commission «maintient l'obligation faite aux assureurs de contracter avec tous les fournisseurs de soins».

Est-ce donc trop demander aux appareils des partis dits «bourgeois» de faire eux aussi confiance à notre engagement et de passer à des projets d'avenir, plutôt que d'en rester à des pseudo-solutions ... du siècle dernier?!

- Enfin, dans un autre registre, un thème de plus en plus utilisé pour faire miroiter une diminution des coûts est celui de la «responsabilité individuelle»: si vous fumez, que vous buvez, que vous shootez, et que vous en attrapez hépatite, cancer, cirrhose ou sida, vous devrez en assumer les conséquences, vous l'avez bien voulu et ça n'est pas à la collectivité de payer pour vous.

On entre là dans une logique, économique, qui a tous les aspects du bon-sens, mais qui ne peut pas être la logique de celles et ceux qui, comme nous, sont en contact avec la réalité concrète: d'abord, derrière des comportements à risque évidents, comme les toxicomanies, ou même avant ces comportements à risque, il y a très souvent des problèmes médicaux qui échappent à toute morale. Franchement, choisit-on de tomber malade? Mais surtout, au nom d'hypothétiques économies et en rendant patients et médecins coupables de coûter - c'est pratique, les boucs émissaires! -, on entre dans un système de «patate chaude» où les coûts ne sont que transférés et qui va rendre les soins et la relation thérapeutique impossibles: le refus de financement des besoins vitaux pour les requérants d'asile montre déjà combien ce genre de «solutions» est pervers et illusoire.

Bref, tout cela montre une fois de plus qu'il y a plusieurs logiques face aux problèmes du système de soin, plusieurs «bons-sens» face à des problèmes difficiles - et qu'ainsi, dans tout ce qui concerne la santé, nous médecins devons absolument faire respecter notre vision médicale et éthique, «hippocratique»: la médecine est décidemment trop importante pour être laissée aux seuls politiciens. 
Autrement dit, il faut le partenariat des médecins pour réformer le système, chacun le sait. C'est de l'arrogance, ou un conservatisme obtus, que d'imaginer le contraire - et il ne suffira pas aux partis et à leurs magistrats de s'abriter derrière deux ou trois «confrères-alibis», fussent-ils des habitués des médias, pour pouvoir imposer des réformes qui font fi de nos principes et de nos exigences légitimes.
Le corps médical a maintenant largement prouvé son ouverture et son sérieux dans son approche des problèmes du système de santé; à nos partenaires aussi, enfin, de s'ouvrir au partenariat!

Jacques de Haller, président de la FMH

Yves Guisan, Conseiller national, vice-président de la FMH

\section{Schluss mit dieser Vergangenheitsromantik!}

Das Thema Gesundheitswesen liegt im Trend: Beide grossen Mitte-Rechts-Parteien unserer nationalen Politlandschaft haben vor zehn Tagen ihre Delegiertenversammlung abgehalten. Dabei drehte sich ein grosser Teil ihrer Traktanden um das Gesundheitswesen.

Es war viel Polemik zu hören, aber leider nichts, was unser Gesundheitswesen einer Lösung näherbringen könnte. Einmal mehr war beeindruckend, wie sehr hinfällige, als überholt und unwirksam bekannte Ideen gewissen Persönlichkeiten als Nährboden dienen. Man ist versucht, ihnen vorzuschlagen, ihre «Powerpoint»-Präsentationen endlich zu ändern ...

Jedenfalls ist es schade, bei alten Ideen zu verharren, während sich die Ärzteschaft - insbesondere via die FMH - bemüht, für zahlreiche Probleme innovative und konstruktive Lösungen vorzuschlagen. Ein paar Beispiele:

- Die Ärzteschaft hat während der gesamten Phase der Kostenneutralität im Anschluss an die Einführung des TARMED bemerkenswerte Anstrengungen unternommen. Diese Bemühungen zeigen, dass die Ärzte selbst in einer schwierigen Situation die erwarteten Ergebnisse erzielen können, und sie lassen für die Zukunft hoffen: Die Leistungs- und Kostenvereinbarungen, die zurzeit als Ablösung der Kostenneutralität in den Kantonen ausgehandelt werden, stehen auf einer guten Ausgangsbasis.

Konnte es der Vorsteher des Eidgenössischen Departements des Innern letzten Samstag deshalb nicht sein lassen, über die partnerschaftlichen Lösungen zu spötteln, weil dies den staatsbürgerlichen Sinn und den guten Willen der Ärzte beweist und die alte Leier von den verantwortungslosen Ärzten Lügen straft?
- Die FMH hat eine ganze Reihe von Vorschlägen unterbreitet, um auf die Anliegen der Politik einzugehen, welche die Qualität ärztlicher Leistungen oder die Wirtschaftlichkeit von Behandlungen betreffen: Wir haben bereits vor einem Jahr gesagt, dass für uns Ärzte zwar das Recht auf einen automatischen Vertragsabschluss bestehenbleiben muss, dieser Automatismus jedoch Bedingungen unterstellt werden kann, die gegenüber der Politik, den Behörden und der Öffentlichkeit sicherstellen, dass Unzulänglichkeiten und Missbräuche wirksam bekämpft und die Kosten unter Kontrolle gebracht werden.

Dies sind ebenfalls partnerschaftliche Vorschläge, sind echte Antworten auf wirkliche Probleme; dank ihnen kann die unergiebige Diskussion über die Aufhebung der freien Arztwahl überwunden werden, die zwangsläufig zu einem Referendum führen würde. Die Kommission für soziale Sicherheit und Gesundheit des Ständerates hat schliesslich sehr wohl begriffen, dass hier eine Gelegenheit beim Schopf gepackt werden musste (siehe Pressemitteilung vom 30. August 2005 über die Spitalfinanzierung): Der Kommissionsentwurf, so schreibt sie, «baut auf dem Vertragszwang zwischen Kassen und Leistungserbringern auf».

Ist es also von den bürgerlichen Parteiapparaten zu viel verlangt, unserem Engagement ebenfalls $\mathrm{zu}$ vertrauen und $\mathrm{zu}$ zukunftsträchtigen Projekten überzugehen, statt bei Pseudolösungen aus dem letzten Jahrhundert zu verharren?

- In einem etwas anderen Register wird ein Thema immer stärker benützt, um eine Kostensenkung vorzuspiegeln: bei der «Ver- 
antwortung des einzelnen». Wenn Sie rauchen, trinken, sich Drogen spritzen und dadurch Hepatitis, Krebs, eine Zirrhose oder Aids bekommen, müssen Sie die Folgen selber tragen, denn Sie haben es ja so gewollt, und es ist nicht Aufgabe der Allgemeinheit, für Sie zu bezahlen.

Wir haben es hier mit einem rein wirtschaftlichen, vernunftorientierten Denken zu tun, das nicht die Logik der Menschen sein kann, die - wie wir - die Realität kennen: Hinter einem offensichtlichen Risikoverhalten wie einer Suchtbefriedigung beziehungsweise schon vorher stehen oft medizinische Probleme, die sich jeglicher moralischer Überlegung entziehen. Wer entscheidet sich denn schon dafür, krank zu werden?

Vor allem aber wird im Namen hypothetischer Einsparungen und durch das Wecken von Schuldgefühlen bei Patienten und Ärzten (weil sie etwas kosten und Sündenböcke ja so praktisch sind!) eine Politik der «heissen Kartoffel» verfolgt, in der die Kosten nur abgewälzt werden und welche die Pflege und die therapeutische Beziehung verunmöglicht: Die Weigerung, die lebensnotwendigen Bedürfnisse von Asylbewerbern zu finanzieren, zeigt bereits, wie pervers und illusorisch solche «Lösungen» sind.
Kurz: All dies beweist einmal mehr, dass es bei den schwierigen Problemen im Gesundheitswesen verschiedene Logiken und Auffassungen von «gesundem Menschenverstand» gibt. Deshalb müssen wir Ärzte bei allem, was die Gesundheit betrifft, unbedingt unsere medizinische und ethische, «hippokratische» Sichtweise geltend machen: Die Medizin ist gewiss zu wichtig, als dass man sie allein den Politikern überlassen könnte.

Oder anders ausgedrückt: Es braucht die Partnerschaft mit den Ärzten, um das System zu reformieren, und das wissen auch alle. Sich das Gegenteil vorzustellen, zeugt von Arroganz oder sturem Konservativismus. Die Parteien und ihre Magistraten können sich nicht einfach hinter zwei oder drei - selbst medienerprobten - «Alibikollegen» verstecken, um Reformen durchzusetzen, die unseren Grundsätzen und legitimen Forderungen widersprechen.

Die Ärzteschaft hat ihre offene Haltung und Ernsthaftigkeit jetzt seit einiger Zeit bewiesen, wenn es um die Lösung von Problemen des Gesundheitswesens ging. Nun sind unsere Partner am Zug, sich dieser Partnerschaft zu öffnen!

Jacques de Haller, Präsident der FMH

Yves Guisan, Nationalrat, Vizepräsident der FMH 